\title{
Caractérisations Structurale, Floristique Et Biomasse Ligneuse Du Peuplement À Hagenia abyssinica Du Parc National De Kahuzi-Biega (Rift Albertin, RDC)
}

\section{Imani Mugisho Gérard, Doctorant}

Université Officielle de Bukavu, Faculté des Sciences et Sciences Appliquées, Laboratoire d'Ecologie et Gestion des Ressources Végétales, Bukavu, RDC et Université de Kisangani, Faculté de Sciences, Laboratoire d’Ecologie et Aménagement, Kisangani

\section{Zapfack Louis, Professeur}

Université de Yaoundé 1, Département de Biologie et Physiologie Végétales, Laboratoire de Systématique et d'Ecologies Végétales, Yaoundé, Cameroun

Mangambu Mokoso Jean De Dieu, Professeur

Université Officielle de Bukavu,

Faculté des Sciences et Sciences Appliquées,

Laboratoire de Systématique Végétale et Biodiversité, Bukavu, RDC

\section{Penedimanja Pancrace, Master étudiant}

Université Officielle de Bukavu,

Faculté des Sciences et Sciences Appliquées, Laboratoire d'Ecologie et Gestion des Ressources végétales, Bukavu, RDC

\section{Mwanga Mwanga Ithe Jean-Claude, Chercheur}

Centre de Recherche en Sciences Naturelles,

Département de Biologie, Laboratoire de Taxonomie Végétale

Boyemba Faustin, Professeur

Université de Kisangani, Faculté de Sciences,

Laboratoire d'Ecologie et Aménagement, Kisangani

\section{Amani Christian, Professeur}

Université Officielle de Bukavu,

Faculté des Sciences et Sciences Appliquées, Laboratoire d'Ecologie et

Gestion des Ressources Végétales, Bukavu, Chercheur CIFOR

\section{Abstract}

Hagenia abyssinica is a characteristic species of mountain forests in the Albertine Rift. In Kahuzi Biega National Park, the species is found between 2000 and $2600 \mathrm{~m}$ altitude. This study intended to characterize Hagenia monospecific forest by providing an account for its floristic 
composition, structure and biomass. Data were collected from 9 different plots of 1 hectare each located in 3 different sites (Tshivanga 2000-2250m asl; Mugabe and Kasirusiru 2400-2600m asl). Parameters including tree diameter $\geq 10 \mathrm{~cm}$, height, dead individuals and young shoots of Hagenia abyssinica were wetting the plot.

The results showed that species richness was significantly low, varying between 8-24 species ( $p<0.05$, Df $=35$ ); while the specific density was 565 \pm 121 individuals. The Richness decreased with the increment of the species density of Hagenia.

The similarity analysis revealed the influence/impact of the site/altitude on the species composition. The diametric structure showed a regressive dynamism within the population found between 2000 and $2200 \mathrm{~m}$ altitude, in which Hagenia specimens were replaced by the specimens of secondary forest species such as Sapium ellipticum, Macaranga neomilbraediana, Neoboutonia macrocalyx. Whereas, between 2400-2600 m altitude, there was a progressive dynamism with several Hagenia young shoots, with no mortality $\left(\chi^{2}=487, p<0.001\right)$.

The average woody biomass was estimated at 108t / ha. This increased as Hagenia density decreased and vegetation became heterogeneous. Human disturbance and climate change effects in the region, could be the causes of variability observed in this population in the Kahuzi-Biega National Park.

Keywords: Kahuzi-Biega National Park, structure, diversity, biomass, Hagenia abyssinica

\section{Résumé}

Hagenia abyssinica est une espèce caractéristique des forêts de montagne dans le Rift albertin. Au Parc National de Kahuzi Biega, l'espèce est présente entre 2000 et 2600m d'altitude. Cette étude s'intéresse à la caractérisation du peuplement pour analyser sa composition floristique, sa structure et en estimer la biomasse selon le caractère du peuplement. Les données ont été collectées dans 9 parcelles de 1ha chacune situées dans trois sites différents (Tshivanga 2000-2250m d'altitude ; Mugaba et Kasirusiru 2400-2600m d'altitude). Il s'agit des données sur le diamètre des arbres $\geq$ $10 \mathrm{~cm}$, sur leur hauteur, les individus morts et les jeunes pousses de Hagenia abyssinica au sein de la parcelle.

Les résultats montrent que la richesse spécifique est faible et varie entre 8 à 24 espèces ( $p$ value $=0,03$; $\mathrm{Df}=35$ ) pendant que la densité spécifique est de $565 \pm 121$ individus. La richesse spécifique diminue lorsque la densité de Hagenia abyssinica devient importante.

L’analyse de similarité indique que le site influence la ressemblance entre les communautés. La structure diamétrique montre que le peuplement situé 
entre 2000 et 2200m d'altitude est en dynamique régressif. Les pieds de Hagenia sont remplacés par les espèces de forêts secondaires comme Sapium ellipticum, Macaranga neomilbraediana, Neoboutonia macrocalyx,... tandis qu'entre 2400-2600m, on a un dynamisme progressif avec plusieurs jeunes pousses de Hagenia et aucune mortalité $\left(\chi^{2}=487 ; \mathrm{p}<0.001\right)$.

La biomasse ligneuse moyenne est de 108t/ha. Elle augmente lorsque la densité de Hagenia diminue et que la végétation devient hétérogène. Les perturbations anthropiques et les effets des changements climatiques dans la région, pourraient être les causes des variabilités observées dans ce peuplement au sein du Parc National de Kahuzi Biega.

Mots clés : PNKB, structure, diversité, biomasse ligneuse, Hagenia abyssinica

\section{INTRODUCTION}

Le regroupement végétal est influencé par le gradient altitudinal en région de montagne (Lovett et al., 2006). Sur ce gradient, plusieurs communautés végétales peuvent se suivre selon le contexte de chaque région, la nature du sol, de la roche mère, de la structure végétale (Ren et al., 2006). Le groupement à Hagenia abyssinica fait partie des formations caractéristiques des régions de montagne dans le Rift albertin (Bussmann, 2006). Au-delà d'une importante diversité, et comme les autres forêts, les écosystèmes montagneux assurent la régulation du climat en jouant sur les facteurs tels que la pluviométrie, l'humidité atmosphérique (Léonard et Oswold, 1996), le stockage de carbone (Yuanying et al., 2008) dans sa biomasse aérienne, permettant de lutter contre les effets négatifs des gaz dit à effet de serre, notamment le $\mathrm{CO}_{2}$. Ainsi, les forêts sont considérées comme les dispositifs efficaces de lutte contre le changement climatique (GIEC, 2006; Kurz et al., 2008; Baccini et al., 2008; Lewis et al., 2009) en constituant des puits actifs (Laporte et al., 2010; Pan et al., 2011) lorsqu'elles ne sont pas perturbées. Toutefois, chaque communauté végétale, accumule la biomasse ligneuse suivant sa composition floristique et sa structure (Henry et al., 2010; Djomo et al., 2010).

Le Parc National de Kahuzi-Biega (PNKB) se trouvant à l'Est de la RDC, se situe au carrefour des centres d'endémismes Guinéo-Congolais et Afro-montagnard (Plumptre et al., 2007). Il abrite plusieurs communautés végétales sous influence d'un gradient altitudinal, comme les autres formations de montagne (Mutke et al., 2011). Parmi ces communautés, on retrouve les groupements de Hagenia abyssinica. En général, ce groupement se situe entre 1800 et $3400 \mathrm{~m}$ d'altitude dans la région afro-montagnarde (White, 1993; Seburanga et al., 2014) et entre 2000 et $2600 \mathrm{~m}$ dans le contexte du PNKB (Pierlot, 1966). 
Hagenia abyssinica est un arbre endémique de la région afromontagnarde (Léonard et Oswold, 1996) et sa population est, particulièrement, considérée en danger d'extinction (Feyissa et al., 2007). Elle est associée à une biodiversité tellement riche et unique si aucune menace n'affecte son dynamisme normal. La structure de son appareil floral pourrait expliquer la complexité de son groupement dans les forêts de montagne. Elle est parmi les rares espèces d'arbres dioïques, mais également l'une de peu d'espèces d'arbres des forêts tropicales humides dont la pollinisation et la dispersion des graines est assurée par le vent (Turneur, 2001; Vamosi et Vamosi, 2005). Dans le Parc National de Kahuzi Biega (PNKB), la structure et la composition de ce groupement n'est pas encore bien connue. On le présente comme caractéristique des horizons moyen et supérieur de la forêt de montagne au PNKB entre 2000 et 2600 m d'altitude (Fischer, 1996; Kabonyi, 2012). La formation de Hagenia abyssinica est sensible aux changements climatiques. Lorsque le climat est favorable à son développement, avec une baisse de la température, cette espèce migre vers les altitudes inférieures du PNKB (Kabonyi, 2012). Ce groupement peut se présenter comme mono dominant avec des vieux arbres ou parfois hétérogène avec d'autres espèces des formations secondaires comme Macaranga neomilbraediana, Sapium ellipticum, Maesa lanceolata, etc.

C'est dans ces perspectives de la variabilité dans la composition, la structure de ce groupement, de son importance dans les écosystèmes de montagne au PNKB ainsi que des enjeux liés aux influences des changements climatiques sur la répartition des espèces, que ce travail est réalisé. Le but est de caractériser le groupement pour ce qui est de sa structure, de sa composition et de mettre en évidence sa contribution dans l'accumulation de la biomasse ligneuse.

\section{MATERIELS ET METHODE Milieu d'étude}

Le présent travail a été réalisé au Parc National de Kahuzi-Biega, particulièrement dans sa partie haute altitude et plus spécifiquement les secteurs de Tshivanga, Mugaba et Kasirusiru (Figure 1). Le Parc national de Kahuzi-Biega est situé à l'Ouest du lac Kivu près de Bukavu, principalement dans la province du Sud-Kivu mais aussi au Nord-Kivu et au Maniema avec sa partie septentrionale. Ce parc national se situe à cheval sur l'Albertine Rift et le bassin du Congo (Fischer, 1993), et il est un habitat exceptionnel pour la protection de la forêt tropicale et les gorilles des plaines orientales, Gorilla berengei graueri. Il se localise entre $27^{\circ} 33^{\prime}$ et $28^{\circ} 46^{\prime}$ de longitude Est et entre $1^{\circ} 36^{\prime}$ et $2^{\circ} 37^{\prime}$ de latitude Sud à l'Ouest du Lac Kivu (Fischer, 1996). 


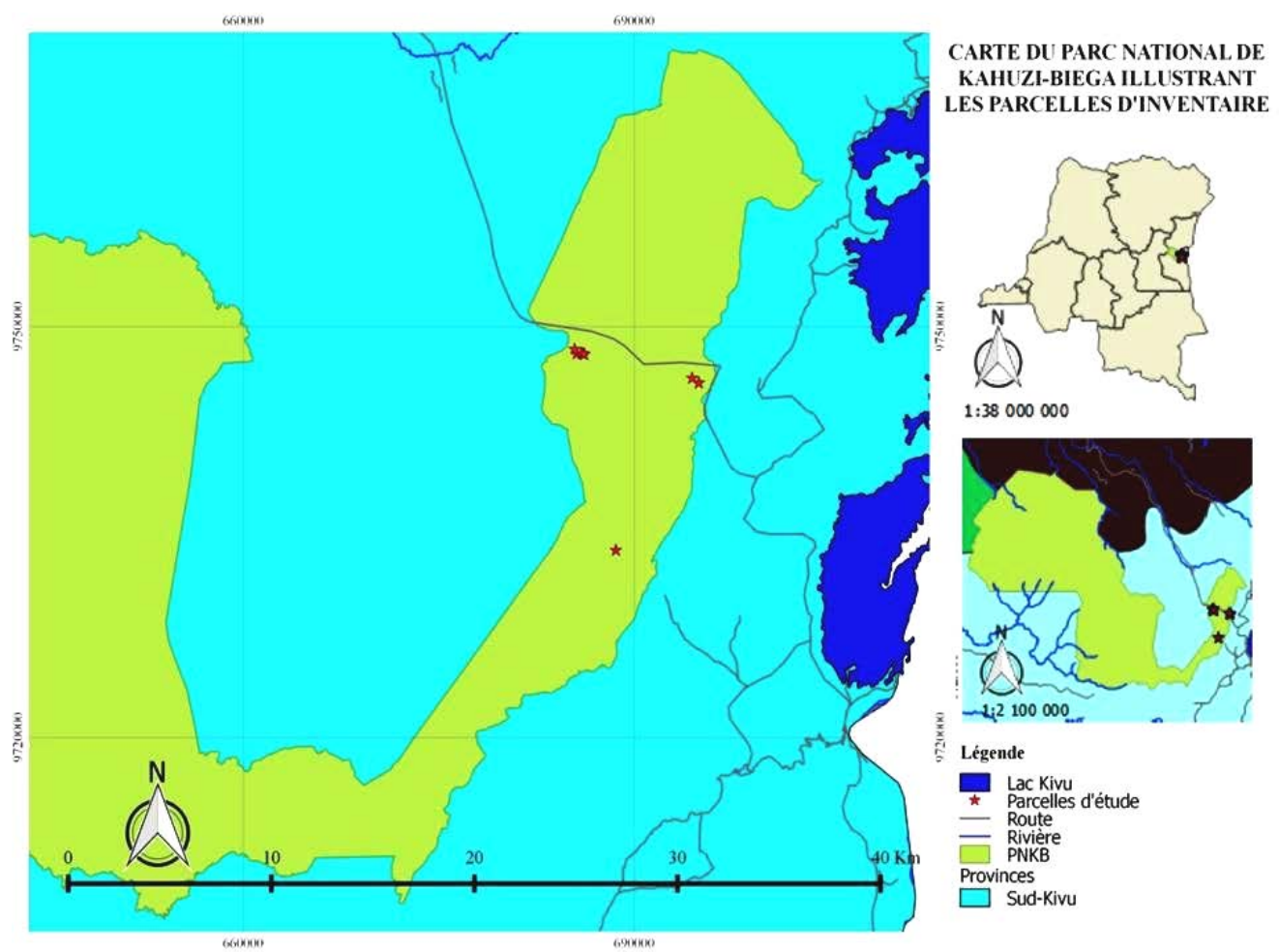

Figure 1: Carte du Parc National de Kahuzi-Biega illustrant les parcelles d'étude en haute altitude

Les précipitations sont très élevées, la moyenne annuelle est d'environ $1619 \mathrm{~mm}$ mais pas distribuées uniformément tout le long de l'année (Yamagiwa et al., 2005). On assiste plutôt à deux saisons des pluies séparées par deux saisons sèches : l'une aux mois de Mai à Juin et l'autre aux mois d'Octobre à Décembre (Fischer, 1993). A cause de la topographie très variée du $\mathrm{PNKB}$, la température moyenne dans la partie haute altitude est d'environ $18^{\circ} \mathrm{C}$ (Fischer, 1996) mais dans le couloir, elle varie de $19^{\circ}$ à $22^{\circ} \mathrm{C}$. Le climat peut être considéré comme uniformément chaud, aussi bien dans le courant d'une journée que annuellement (Yamagiwa et al., 2005).

\section{Méthode d'étude}

\section{Collecte de données}

En effet, 9 parcelles, d'1ha chacune, semi permanentes de $100 \mathrm{~m}$ x100 m chacune, ont été installées dans trois sites de la partie haute altitude du PNKB (Mugaba, Tshivanga et Kasirusiru). Ainsi, 3 parcelles à Tshivanga, 5 à Mugaba et 1 à Kasirusiru suivant la disponibilité de la formation et le respect de la distance d'au moins 500m entre les parcelles. Les parcelles ont été subdivisées en 4 placettes pour faciliter les inventaires. 
Pour les espèces ligneuses, à l'aide d'un DBH mètre, on a pris le diamètre à la hauteur de la poitrine des arbres (à 1,30 m au-dessus du sol) des espèces à $\mathrm{DBH} \geq 10 \mathrm{~cm}$, à l'exception de celles présentant des déformations soit naturelles ou pathologiques, dues au stress. Pour ces derniers, la mesure s'effectuait au-dessus ou en dessous du défaut. La hauteur a été mesurée avec un Laser ACE pour quelques pieds de différentes espèces et une équation de régression générée a permis d'avoir la hauteur de tous les arbres à l'hectare (Feldpausch et al., 2012).

Pour évaluer la régénération et la mortalité, toutes les jeunes pousses (individus à $\mathrm{DBH}<10 \mathrm{~cm}$ ) de Hagenia abyssinica ainsi que ses individus morts ont été comptés.

L'estimation de la biomasse des arbres inventoriés s'est faite en utilisant les équations allométriques (Chave et al., 2014). On a retenu l'équation reposant sur trois variables dendrométriques à savoir la hauteur (m), le diamètre $(\mathrm{cm})$ et la densité des arbres (Zanne et al., 2009) ce qui minimise l'erreur d'estimation de la biomasse (Kearsley et al., 2013).

\section{Analyse de données floristiques \\ Richesse et abondance spécifiques}

La richesse spécifique correspond au nombre d'espèces recensées à l'échelle de la parcelle et l'abondance spécifique le nombre d'individus à la parcelle.

La surface terrière

La surface terrière a été calculée suivant la formule suivante :

$\mathrm{S} . \mathrm{T}=\pi D^{2} / 4$ avec $\mathrm{D}$ diamètre des arbres et $\pi=3,14$.

Densité relative d'une espèce

La densité relative a été calculée suivant la formule :

D.R $=\frac{\text { Nombre } d^{\prime} \text { individus d'une espèce }}{\text { Nombre total } d^{\prime} \text { individus dans l'échantillon }} \times 100$

L'indice de Pièlou

Cet indice varie de 0 à 1 et permet de savoir comment les individus se répartissent entre les espèces à la parcelle. Il se calcule par la formule suivante : $J=H^{\prime} / \log _{2} S$ Avec S nombre total d'espèces et $\mathrm{H}^{\prime}$ indice de Shannon.

\section{Tests statistiques}

L'Analyse de la Variance (Anova) a permis de connaitre la variabilité de la diversité et de la biomasse entre les parcelles au seuil de $5 \%$ en considérant les parcelles et leurs placettes ou quadra. Les espèces caractéristiques de chaque groupe ont été déterminées par l'approche IndVal (De Cáceres, 2013). A chaque espèce caractéristique, sont ajoutées sa 
probabilité de fidélité (appartenance exclusive au groupe) et d’occurrence (fréquence de l'espèce au sein du groupe). Pour déterminer la variabilité de la structure de diamètre entre les communautés l'analyse de Chi carré a été appliquée. L’Analyse de la similarité de Jaccard a permis de mettre en évidence la ressemblance entre les communautés étudiées. Les tests de corrélation ont été faits les relations entre la densité en Hagenia abyssinica et la quantité de la biomasse à la parcelle. Toutes les analyses ont été faites sous le logiciel R.

\section{RESULTATS \\ Diversité et composition floristique}

La richesse spécifique est de 8 à 24 espèces à la parcelle. Le nombre d’individus moyen 565 \pm 121 (soit 378 à 773 individus par parcelle). L'indice de pièlou oscille entre 0,57 et 0,79. La richesse spécifique varie de manière significative entre les différentes parcelles ( $p$ value $=0,03 ; \mathrm{Df}=35$ ) alors que l'abondance spécifique ne varie pas ( $\mathrm{p}$ value $=0.08 ; \mathrm{Df}=35)$ tout comme l'indice de Pièlou ( $\mathrm{p}=0.15$; $\mathrm{Df}=35)$. Par ailleurs, la densité relative de Hagenia abyssinica varie de 22,5\% à 61,5\% (Tableau 1).

Tableau 1: Variation de la diversité floristique dans les parcelles d'étude

\begin{tabular}{|lllll|}
\hline Parcelles & $\begin{array}{l}\text { Richesse } \\
\text { spécifique }\end{array}$ & Abondance & $\begin{array}{l}\text { Indice } \\
\text { Pielou }\end{array}$ & $\begin{array}{l}\text { de } \\
\text { H. abyssinica }(\%)\end{array}$ \\
\hline P1 & 19 & 773 & 0,66 & 38,29 \\
P2 & 21 & 652 & 0,72 & 29,10 \\
P3 & 24 & 609 & 0,79 & 22,50 \\
P4 & 12 & 506 & 0,57 & 58,10 \\
P5 & 13 & 497 & 0,58 & 60,56 \\
P6 & 10 & 468 & 0,64 & 61,54 \\
P7 & 13 & 533 & 0,69 & 49,93 \\
P8 & 10 & 670 & 0,63 & 56,87 \\
P9 & 8 & 378 & 0,73 & 51,06 \\
\hline
\end{tabular}

Le pourcentage important de Hagenia abyssinica à la parcelle indique le caractère monodominant ou hétérogène du groupement.

Certaines communautés se ressemblent au seuil de 50\%. En effet, les parcelles installées à Tshivanga sont très proches (56\%) entre elles et constituent le premier groupe; il s'agit des parcelles P1_Tshivanga, P2_Tshivanga et P3_Tshivanga. Le deuxième groupe à ce même seuil est constitué de toutes les parcelles installées à Mugaba (environ 60\%); il s’agit des parcelles P4_Mugaba, P5_Mugaba, P7_Mugaba, P8_Mugaba. La parcelle installée à Kasirusiru P9_Kasirusiru est plus proche de la parcelle P6_Mugaba à environ 48 \% et constitue un autre groupe (figure 2). 


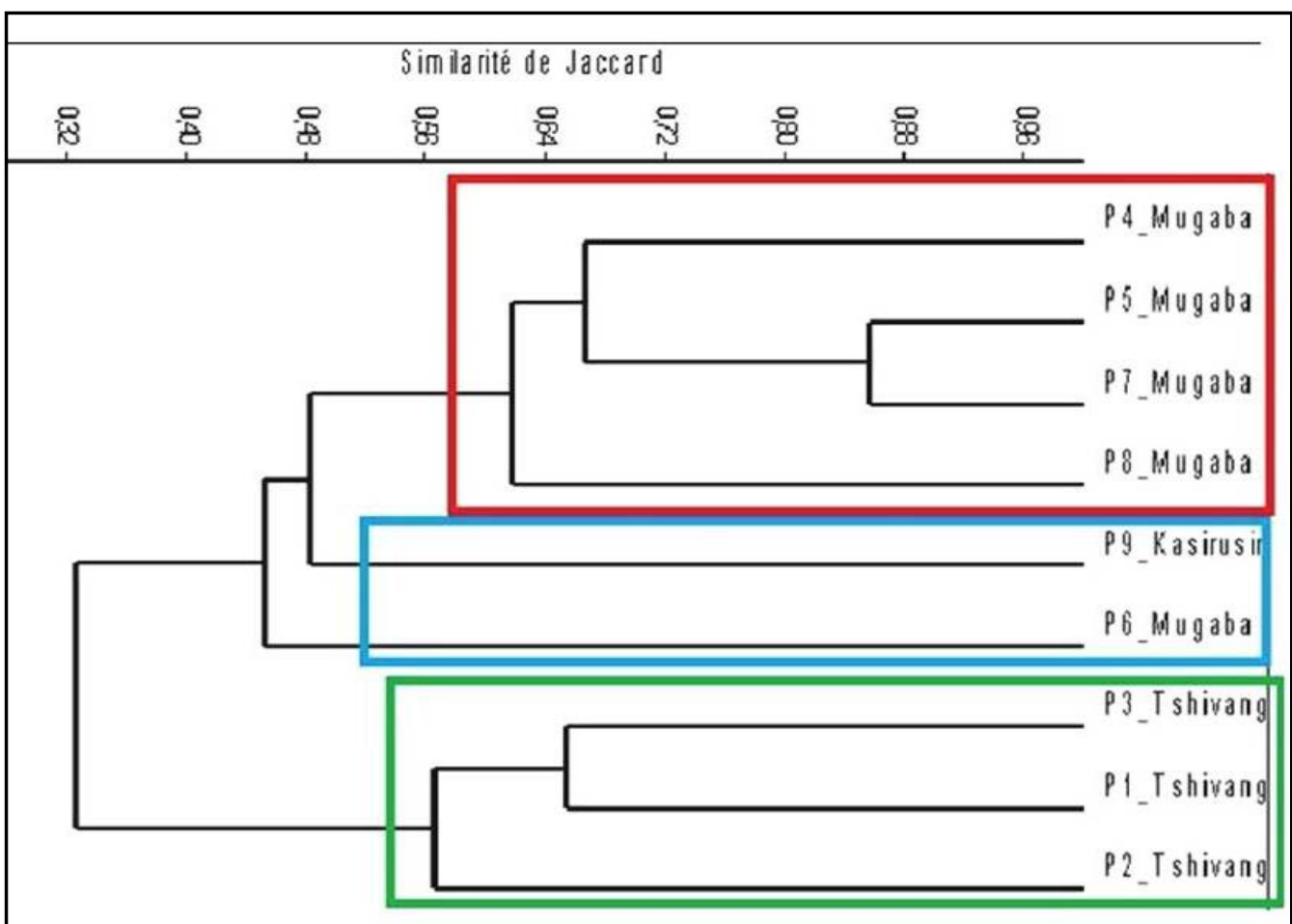

Figure 2: Dendrogramme de similarité en fonction de la présence spécifique (Indice de Jaccard). Trois communautés différentes se constituent en raison d'une ressemblance de 50\% basée sur la présence absence des espèces à l'échelle de la parcelle.

Chaque groupe est caractérisé par certain nombre d’espèces (tableau 2). On distingue les espèces du groupe de Tshivanga, le groupe de Mugaba et le groupe Kasirusiru-Mugaba.

Tableau 2: Espèces indicatrices ou caractéristiques de chaque groupe

\begin{tabular}{|c|c|c|c|c|c|}
\hline \multirow[t]{2}{*}{ Num } & \multirow[t]{2}{*}{ Espèces } & \multicolumn{2}{|c|}{ Probabilité } & \multicolumn{2}{|c|}{ Indival (IndVal.g) } \\
\hline & & $\begin{array}{l}\text { A } \\
\text { (Fidélité) }\end{array}$ & $\begin{array}{l}\text { B } \\
\text { (Occurrence } \\
\text { ) }\end{array}$ & Indival & $p>5 \%$ \\
\hline & \multicolumn{5}{|c|}{ Espèces indicatrices du groupe de Tshivanga } \\
\hline 1 & Allophyllus kiwuensis & 1.0000 & 1.0000 & 1.000 & $0.014^{*}$ \\
\hline 2 & Bridelia micrantha & 1.0000 & 1.0000 & 1.000 & $0.014^{*}$ \\
\hline 3 & Neoboutonia macrocalyx & 1.0000 & 1.0000 & 1.000 & $0.014^{*}$ \\
\hline 4 & Sapium ellipticum & 1.0000 & 1.0000 & 1.000 & $0.014 *$ \\
\hline 5 & Tabernaemontana johnstonii & 1.0000 & 1.0000 & 1.000 & $0.014^{*}$ \\
\hline \multirow[t]{2}{*}{6} & Galiniera coffeoides & 0.955 & 1.0000 & 0.977 & $0.021 *$ \\
\hline & \multicolumn{5}{|c|}{ Espèces indicatrices du groupe de Mugaba } \\
\hline 1 & Syzygium guineense & 1.0000 & 1.0000 & 1.000 & $0.037 *$ \\
\hline \multirow[t]{2}{*}{2} & Polyscias fulva & 0.984 & 1.0000 & 0.992 & $0.012 *$ \\
\hline & \multicolumn{5}{|c|}{ Espèces indicatrices du groupe de Kasirusiru-Mugaba } \\
\hline 1 & Agauria salicifolia & 0.994 & 1.0000 & 0.997 & $0.044^{*}$ \\
\hline
\end{tabular}


En effet, les espèces caractéristiques varient selon le site, même si on reste dans le même groupement. A Tshivanga Allophyllus kiwuensis, Bridelia micrantha, Neoboutonia macrocalyx, Sapium ellipticum, Tabernaemontana johnstonii caractérisent la strate arborescente et Galiniera coffeoides le sous-bois. A Mugaba, Syzygium guineense, Polyscias fulva, caractérisent la strate arborescente. Pour le troisième groupe (Kasirusiru, Mugaba), Agauria salicifolia est l'espèce arbustive caractéristique.

\section{Structure de la végétation}

\section{Structure horizontale et classe de diamètre}

Les quatre espèces écologiquement importantes de l'ensemble du groupement étudié (IVI>10, Tableau 3) de la partie haute altitude du PNKB sont principalement Hagenia abyssinica, Macaranga neomildbraediana, Rapanea melanophloeos et Agauria salicifolia.

Tableau 3: Structure des essences ligneuses sur l'ensemble de parcelles d'étude

\begin{tabular}{|c|c|c|c|c|c|c|}
\hline Espèces & $\begin{array}{l}\text { Nbre Indi } \\
\text { total }\end{array}$ & $\begin{array}{l}\text { DBH total } \\
\text { en } \mathrm{cm}\end{array}$ & DoR & DR & Freq & IVI \\
\hline Hagenia abys & 2343 & 58135 & 87,15 & 46,07 & 6,98 & 140,20 \\
\hline Macaranga neomildbraediana & 993 & 19718,5 & 10,03 & 19,52 & 6,98 & 36,53 \\
\hline Rapanea melanophloeos & 377 & 5042 & 0,66 & 7,41 & 6,20 & 14,27 \\
\hline Agauria salicifolia & 374 & 7435 & 1,43 & 7,35 & 5,43 & 14,21 \\
\hline
\end{tabular}

Légende: Nombre d'individus (Ni), Diamètre à la hauteur de la poitrine (DBH ou DHP); Surface Terrière (ST); Dominance relative (DoR), Fréquence relative (Freq) et Indice de valeur d'Importance écologique (IVI)

Pour mieux comparer la structure de diamètre et suivant la taille des arbres étudiés, quatre classes de diamètre ont été constituées. Le plus grand diamètre a été retrouvé au site de Tshivanga chez Hagenia abyssinica. Sur l'ensemble de la communauté, la distribution suit une succession progressive avec une structure en $\mathrm{J}$ inversé, typique des forêts tropicales non perturbées. Les classes de diamètre inférieures ont plus d'individus que les classes supérieures (Figure 3). Entre les différentes parcelles, la structure diamétrique est variable $\left(\chi^{2}=253,01 ; \mathrm{p}<0.001\right)$.

L'analyse des contributions de chaque classe de diamètre à la statistique $\chi^{2}$ totale (tableau 4) montre que, dans les classes $10-20 \mathrm{~cm}, 20-30$ $\mathrm{cm}$ et $30-40 \mathrm{~cm}$, sont plus contributives pour l'ensemble des tranches d'étude. 


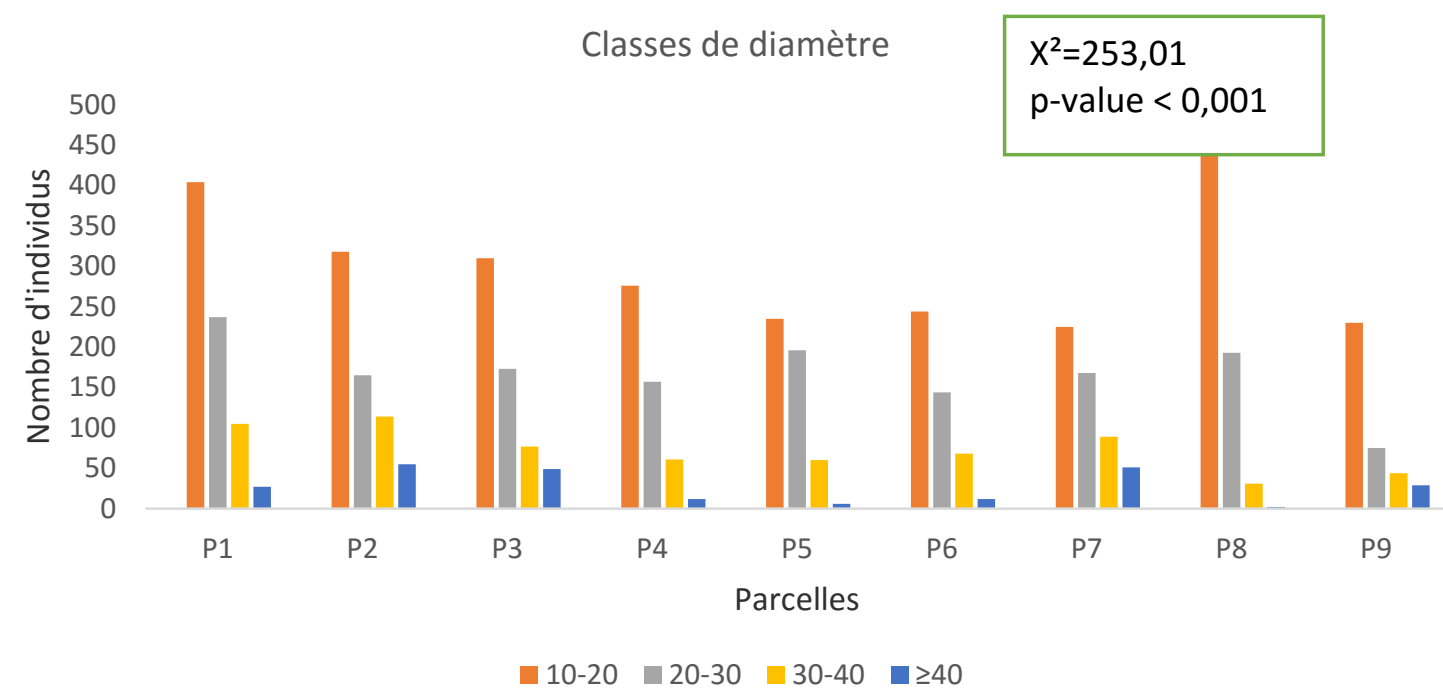

Figure 3 : Distribution diamétrique des tiges dans chaque parcelle étudiée en considérant toutes les espèces de la parcelle. Légende: P1, P2 ...= Parcelle 1, Parcelle $2, \ldots$

Tableau 4: Contribution (en \%) des classes de diamètre à la statistique de chi carré (l'interprétation du tableau se fait par ligne)

\begin{tabular}{|l|l|l|l|l|}
\hline Parcelles & $\mathbf{1 0 - 2 0}$ & $\mathbf{2 0 - 3 0}$ & $\mathbf{3 0 - 4 0}$ & $\geq \mathbf{4 0}$ \\
\hline P1 & 52,8 & 30,7 & 13,6 & 3,5 \\
P2 & 48,8 & 25,3 & 17,5 & 8,4 \\
P3 & 50,9 & 28,4 & 12,6 & 8 \\
P4 & 54,5 & 31 & 12,1 & 2,4 \\
P5 & 47,3 & 39,4 & 12,1 & 1,2 \\
P6 & 52,1 & 30,8 & 14,5 & 2,6 \\
P7 & 42,2 & 31,5 & 16,7 & 9,6 \\
P8 & 66,3 & 28,8 & 4,6 & 0,3 \\
P9 & 60,8 & 19,8 & 11,6 & 7,7 \\
\hline
\end{tabular}

\section{Aspect de la dynamique au sein de groupement à Hagenia abyssinica}

L'analyse de la distribution de diamètre des individus de Hagenia abyssinica dans les différents sites, montre que pour les parcelles se trouvant dans le site de Tshivanga, l'espèce est dans une dynamique de régression car le nombre d'individus dans les classes supérieures sont nombreux par rapport à ceux des classes inférieures. Pour les parcelles de Mugaba et de Kasirusiru, la succession est progressive, la structure en J inversé (Figure 4; Tableau 5). 


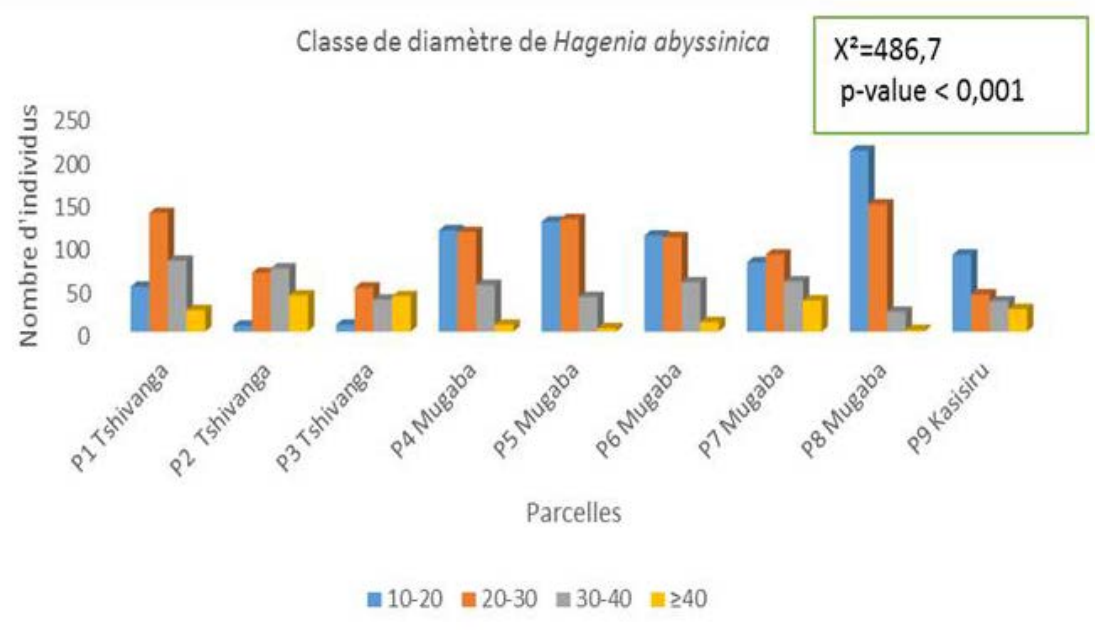

Figure 4: Structure diamétrique des individus de Hagenia abyssinica dans le peuplement

Tableau 5: Contribution (en \%) des classes à la variation de la structure diamétrique spécifique à Hagenia abyssinica

\begin{tabular}{|l|l|l|l|l|}
\hline Parcelles & $10-20$ & $20-30$ & $30-40$ & $\geq 40$ \\
\hline P1 & 17,6 & 46,3 & 27,7 & 8,4 \\
P2 & 3,7 & 35,8 & 38,4 & 22,1 \\
P3 & 5,8 & 37,2 & 27 & 29,9 \\
P4 & 39,8 & 39,1 & 18,4 & 2,7 \\
P5 & 42,2 & 43,2 & 13,3 & 1,3 \\
P6 & 38,5 & 37,8 & 19,8 & 3,8 \\
P7 & 30,4 & 33,8 & 22,1 & 13,5 \\
P8 & 54,9 & 38,6 & 6 & 0,5 \\
P9 & 46,1 & 22,3 & 18,1 & 13,5 \\
\hline
\end{tabular}

Les parcelles situées à Tshivanga ont une mortalité élevée et une régénération nulle. Pour les parcelles de Kasirusiru et Mugaba la mortalité devient faible et la régénération élevée (Tableau 6).

Tableau 6: Dynamique des individus de Hagenia au sein du le peuplement

\begin{tabular}{|llll|}
\hline Parcelles & Individus mort & Individus en régénération & Sites \\
P1 & 80 & 0 & Tshivanga \\
P2 & 93 & 0 & Tshivanga \\
P3 & 38 & 0 & Tshivanga \\
P4 & 17 & 45 & Mugaba \\
P5 & 45 & 32 & Mugaba \\
P6 & 25 & 90 & Mugaba \\
P7 & 34 & 18 & Mugaba \\
P8 & 5 & 31 & Mugaba \\
P9 & 7 & 28 & Kasirusiru \\
Total & 344 & 244 & \\
\hline
\end{tabular}




\section{Estimation de la biomasse ligneuse}

\section{Biomasse par espèce dans le groupement}

La biomasse dans les différentes parcelles est variable $(\mathrm{p}=0.0032$; $\mathrm{Df}=35$ ). Elle varie entre $58,6 \mathrm{t} / \mathrm{ha}$ et $177,9 \mathrm{t} / \mathrm{ha}$; soit $28,11 \mathrm{tC} / \mathrm{ha}$ et $85,4 \mathrm{tC} / \mathrm{ha}$. Les parcelles situées à Tshivanga ont plus de biomasse et de carbone stocké.

La figure 5 illustre les premières espèces ayant la biomasse totale importante sur l'ensemble des parcelles étudiées. Les trois premières en tête sont: $H$. abyssinica, $M$. neomildibraediana et A. salicifolia respectivement avec $637,80 \mathrm{t} / \mathrm{ha}, 126,06 \mathrm{t} / \mathrm{ha}$ et 59,31 t/ha.

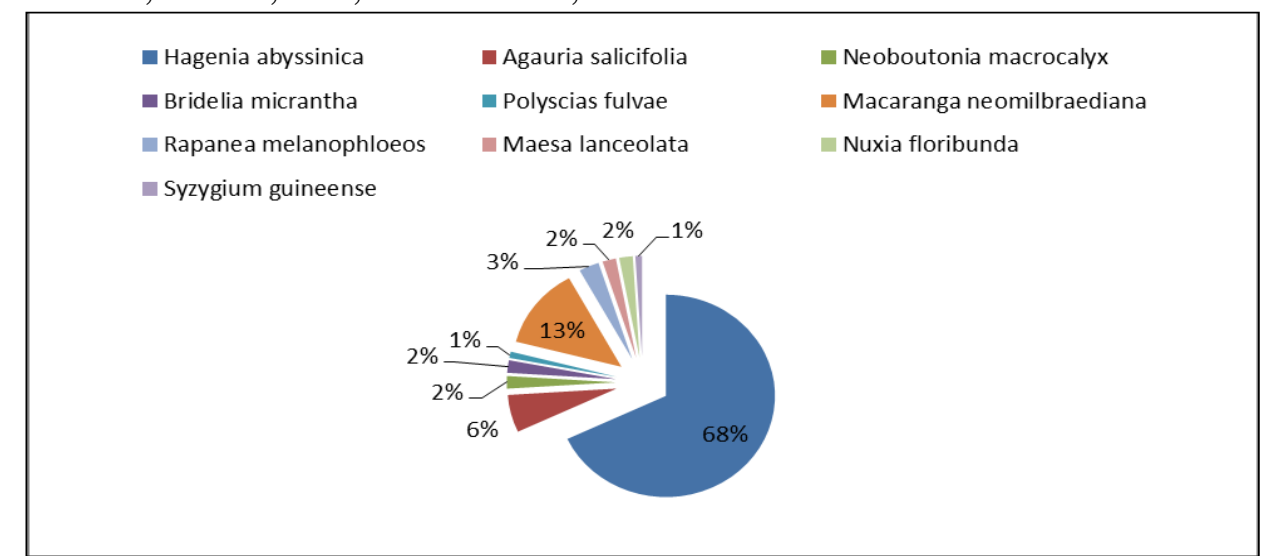

Figure 5: Quantité de biomasse (AGB) par espèce sur l'ensemble du peuplement

\section{Biomasse ligneuse et structure de la végétation}

En analysant la proportion de la biomasse totale pour chaque classe de diamètre dans l'ensemble de parcelles étudiées, il s'observe que la plus grande quantité est dans la classe intermédiaire (Figure 6)

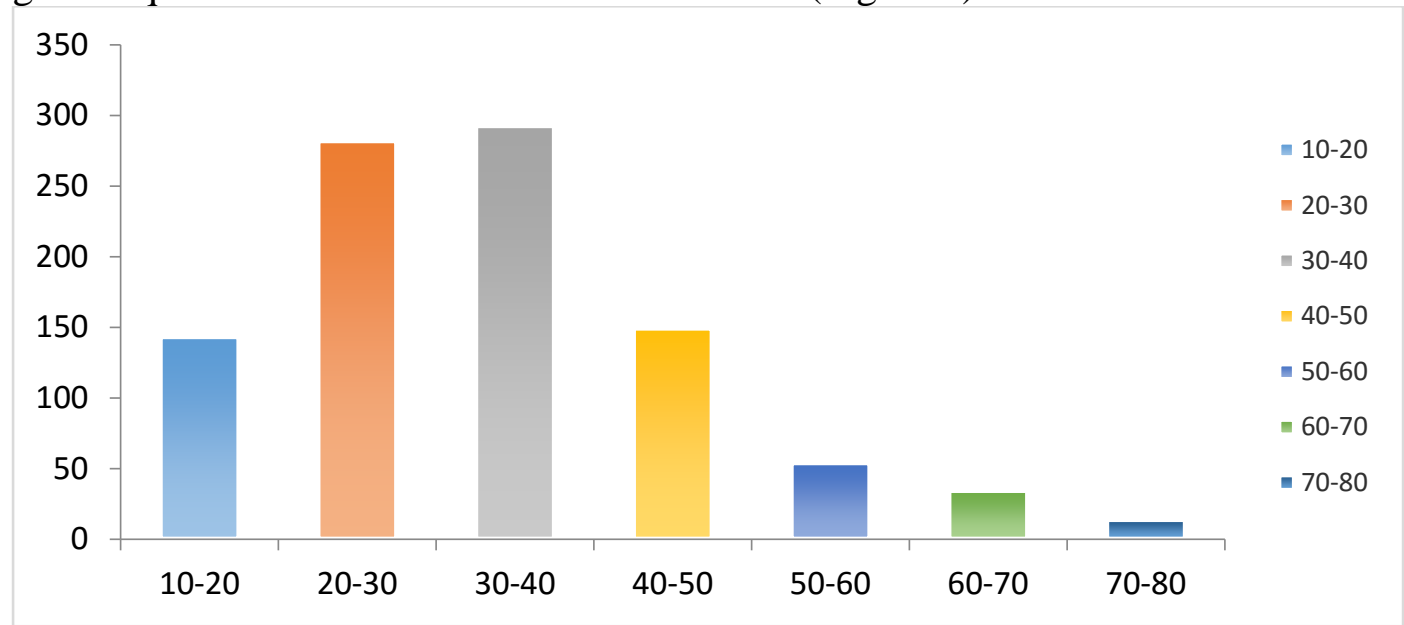

Figure 6 : Quantité de biomasse totale (AGB en t/ha) par classes de diamètre 
En effet, lorsqu'on note une forte densité de Hagenia abyssinica à l'échelle d'une parcelle, la biomasse diminue également $(\mathrm{p}<0.05$ et $\mathrm{r}=$ $0,42)$. Autrement dit il y a une relation négative entre la densité à $H$. abyssinica et l'AGB (Figure 7)

\section{二 Densité Hagenia}

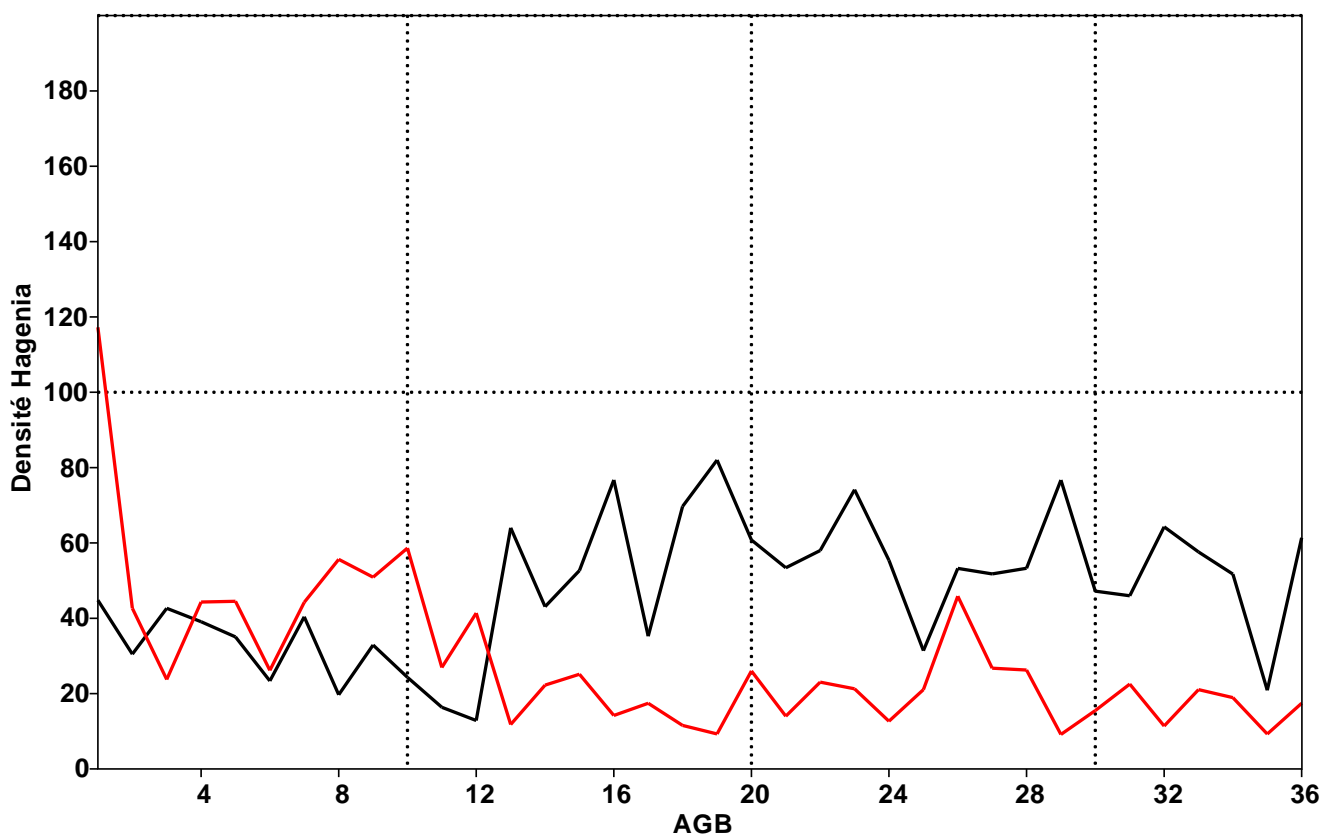

Figure 7: Corrélation entre la biomasse ligneuse (AGB) et la densité de $H$. abyssinica

\section{DISCUSSION}

\section{Variabilité dans la composition et la structure de la végétation du groupement}

Les populations des plantes en milieu des montagnes vivent dans des conditions tellement différentes de celles des continents que plusieurs causes interviennent pour produire une même structure coadaptée, au sein de laquelle il est difficile de dissocier les agents de sélection (Mangambu, 2013). Les organismes ont dû s'adapter aux conditions écologiques et subir des modifications évolutives dont l'ensemble constitue le syndrome d'insularité (Tassin et al., 2004). Une diminution du nombre d'espèces, le nanisme, élargissement des niches, sédentarité, entre autres, peuvent être considérés comme traits caractéristiques. Les résultats de la présente étude prouvent que la diversité floristique varie au sein du peuplement à Hagenia abyssinica du Parc National de Kahuzi Biega (PNKB). En effet, la richesse spécifique est variable entre les parcelles. L'abondance et l'équitabilité de Pièlou ne varient pas. La moyenne d'individu à la parcelle étant de 560 
individus, reconnue pour les régions tropicales en général (Pascal, 2003) bien que cette densité devient faible (450 individus) à certain endroit, en raison peut être des contraintes particulières comme la présence des bambous mélangé aux Hagenia.

La forte densité de $H$. abyssinica s’accompagne également d'une faible diversité floristique (richesse et abondance) au sein d'une parcelle. Cette observation avait été faite dans la région de Kisangani pour le peuplement de Pericopsis elata (Boyemba, 2011) et du Cameroun (Ngueguim, 2013). En effet, pour le peuplement à caractère de monodominance, on note une faiblesse de diversité (Torti et al., 2001). Ceci pourrait être dû à l'influence de Hagenia abyssinica sur la régénération d'autres espèces à la parcelle d'étude. Il a été prouvé que dans les formations monodominantes en région tropicale, les traits structurels de la plante dominante influencent plus son peuplement que la structure du sol (Torti et al., 2001; Peh et al., 2011). Ainsi, la monodominance s'accompagne de la faible diversité dans cette région du Rift congolais.

En se basant sur la valeur écologique des espèces, les espèces importantes sont Hagenia abyssinica, Macaranga neomildibraediana, Rapanea melanophloeos et Agauria salicifolia en raison de leur abondance, dominance et fréquence dans le peuplement. Ces espèces sont caractéristiques de forêt secondaire de la haute altitude du PNKB (Fischer, 1996) et même des formations de montagne dans le Rift en général (Habiyaremye, 1995)

\section{Tendance d'un dynamisme régressif au sein de la population}

Les caractéristiques physionomiques de la végétation reflètent les adaptations des espèces aux conditions environnementales, révélant ainsi la structure de la forêt (Mangambu, 2013). Pour cette étude, la densité des tiges diminue avec l'augmentation des classes de diamètre (Pascal, 2003). En considérant les informations générales à la parcelle, on a constaté que la structure de la classe de diamètre est un J inversé. Les individus diminuent avec l'augmentation des classes de diamètre. Une réalité reconnue pour les arbres et même le peuplement monodominant de la région tropicale (Pascal, 2003; Boyemba, 2011). L’arbre le plus gros est $H$. abyssinica avec $80 \mathrm{~cm}$ de diamètre.

Toutefois, la densité élevée de cette espèce à l'échelle d’une parcelle, ne s'accompagne pas forcement d'une surface terrière importante. Par exemple, le gros diamètre est inventorié à Tshivanga, site avec une faible densité de l'espèce. Ce résultat pourrait expliquer un dynamisme régressif en terme de changement de la formation initiale, mais progressif consistant à remplacer les vieux peuplements de Hagenia par une formation secondaire constituées des espèces comme Allophyllus kiwuensis, Bridelia micrantha, 
Neoboutonia macrocalyx, Sapium ellipticum, Tabernaemontana johnstonii, Macaranga neomilbraediana. Cette dynamique forestière qui s'installe, justifierait aussi la diversité importante observée localement aux sites concernés. Toutefois, cette hypothèse mérite d’être vérifiée en suivant les peuplements à travers les parcelles permanentes.

En se basant sur les classes de diamètre spécifique de $H$. abyssinica, les résultats ont montré que pour les parcelles installées à Tshivanga, la végétation suit une succession régressive alors que la structure pour Kasirusiru et Mugaba est en $\mathrm{J}$ inversé, une succession progressive. Ce qui prouverait une tendance à l'hétérogénéisation de la végétation à certain endroit du Parc où la formation se trouve. Cette situation se confirme par la mortalité et la régénération des individus de $H$. abyssinica. En effet, pour le site de Tshivanga, on note un taux élevé de mortalité (211/344) et zéro taux de régénération (aucune jeune pousse d'individus de $H$. abyssinica). Cependant, pour les sites de Kasirusiru et Mugaba où la dynamique est progressive, on observe une bonne régénération avec plusieurs pousses de $H$. abyssinica et un très faible taux de mortalité (133/344). Dans la région du Rift albertin, il se pose déjà la question sur le statut de cette formation endémique de montagne (Feyissa et al., 2007; Seburanga et al., 2014). D'aucun pense qu'un faible changement de la température dans cette zone (Akonkwa et al., 2015) pourrait influencer la diversité biologique dans sa composition et sa structure.

Le phénomène observé à Tshivanga amène à se questionner tout de même sur les statuts (l'avenir) de ce peuplement. En effet, le remplacement de la formation de $H$. abyssinica par d'autres espèces de formation secondaires devrait avoir un impact sur la conservation et la protection de ce peuplement en haute altitude du PNKB. Comme l'avait indiqué une analyse de la flore du passé de la haute altitude du PNKB, la hausse de la température influence la migration des espèces à la recherche du climat favorable (Kabonyi, 2012) dans les montagnes du PNKB. De même, $H$. abyssinica est une espèce caractéristiques des altitudes supérieures au PNKB au-delà de 2400 m (Imani et al., 2016 Submitted). Ainsi donc, sa disparition en dessous de cette altitude pourrait avoir de relation avec les perturbations du climat signalées dans la région (Akonkwa et al., 2015). Les résultats ont montré que l'espèce continue à régénérer dans les altitudes supérieures (au-delà de $2300 \mathrm{~m}$ ) pendant qu'elle se remplace par les autres espèces de forêts secondaire aux altitudes inférieures à $2300 \mathrm{~m}$. Ceci prouve que l'espèce peut être considérée comme indicateur du changement climatique dans la flore de montagne du PNKB et ses environs. 


\section{La structure et la composition du peuplement influence sa biomasse ligneuse}

La biomasse ligneuse varie selon les communautés végétales (Baccini et al., 2008, Fayolle et al., 2013). On a trouvé la quantité moyenne de la biomasse à l'hectare de 107,8t/ha. Cette valeur s'approche de la quantité moyenne reconnue pour les régions de montagne (GIEC, 2006) sur les altitudes supérieures à $1500 \mathrm{~m}$, soit 169t/ha sur base de la télédétection (Baccini et al., 2008). Cependant, il s’observe une variabilité due à la composition floristique et à la structure de la végétation de sorte que la quantité de biomasse/carbone à l'hectare varie suivant les parcelles.

En effet, lorsque la végétation est de plus en plus hétérogène, faible densité de Hagenia abyssinica, la biomasse à la parcelle devient importante (cas des parcelles installées à Tshivanga) et faible dans le cas d’homogénéité, pour les parcelles à Kasirusiru et Mugaba. Ceci pourrait être dû aux différents paramètres dendrométriques considérés pour l'estimation de la biomasse. Les auteurs ont souligné l'influence majeure de la densité du bois sur l'accumulation de la biomasse d'une espèce (Kearsley et al., 2013) ainsi que d'autres variables comme la richesse et de l'abondance spécifiques (Djuikouo et al., 2010). Lorsque la végétation devient hétérogène, la richesse spécifique augmente et favorise en même temps une hausse de la valeur totale de la densité anatomique des espèces à l'hectare. La biomasse à l'échelle de la parcelle devient ainsi élevée par rapport aux parcelles avec une végétation monodominante de $H$. abyssinica.

\section{CONCLUSION}

L’objectif de cette étude était de caractériser la structure, la composition et estimer la biomasse ligneuse dans un peuplement à Hagenia abyssinica de la haute altitude du Parc National de KahuziBiega. Au terme de ce travail, on retient que la diversité floristique et la composition floristique varie au sein du peuplement étudiée. Aux altitudes inférieures à $2300 \mathrm{~m}$ du Parc, le peuplement devient de plus en plus hétérogène avec une faible densité de Hagenia abyssinica laquelle s’accompagne des espèces caractéristiques comme Tabernaemontana johnstonii, Allophyllus kiwuensis, Bridelia micrantha, Neoboutonia macrocalyx, Sapium ellipticum, Galiniera coffeoides. Dans les altitudes supérieures à $2300 \mathrm{~m}$, le peuplement reste monodominant avec comme espèce caractéristique Syzygium guineense, Polyscias fulvae et Agauria salicifolia. La diversité est élevée lorsque le peuplement est hétérogène. Les espèces ayant une grande valeur d'importance écologique sont respectivement Hagenia abyssinica, Macaranga neomildibraediana, Rapanea melanophloeos, Agauria salicifolia. 
L'analyse de la structure diamétrique indique une régression des individus de Hagenia à Tshivanga (altitude inférieure à $2300 \mathrm{~m}$ ) avec un taux élevée de mortalité de ces individus pendant qu’à Kasirusiru et Mugaba (altitude supérieure à $2300 \mathrm{~m}$ ) on note une succession progressive avec beaucoup d'individus jeunes et âgés de Hagenia et une mortalité presque nulle. Cette situation a été attribuée aux effets des changements climatiques observés actuellement en se basant sur les hypothèses des analyses palynologiques réalisées dans la région.

En ce qui concerne la quantité de la biomasse/carbone, il s'observe une variabilité due à la composition floristique et à la structure de la végétation à la parcelle. La densité du bois, la richesse et l'abondance spécifique influencent l'accumulation de la biomasse dans le groupement étudié pour la partie haute altitude du Parc National de Kahuzi Biega.

Ainsi, le peuplement de Hagenia reste un bon indicateur pour évaluer les effets des changements climatiques dans les forêts de montagne du PNKB. Les parcelles permanentes mériteraient d'être retracées et suivies pour comprendre la dynamique de $H$. abyssinica et tester l'hypothèse de l'influence du climat sur son écologie. Enfin, la conservation du Parc National de Kahuzi-Biega devrait intégrer la politique des changements climatiques pour améliorer les mesures de gestion sur le long terme.

\section{Remerciements}

Le présent travail a été soutenu financièrement par le projet FCCC (Appui à l'UNIKIS: "Forêts et Changement Climatique au Congo") financé par la Commission européenne, mis en œuvre par le CIFOR en partenariat avec l'Université de Kisangani (UNIKIS) et RSD pour le soutien logistique. Les auteurs remercient également tous ceux qui ont contribué à la collecte et au traitement des données.

\section{References:}

Akonkwa, B., Muhigwa, B., Montcho, S.A., Laleye, P. (2015). Climate change and its impact on the fisheries in Lake Kivu , East Africa. Journal of Biodiversity and Ecological Sciences, 6, 312-327.

Baccini, A., Laporte, N., Goetz, S.J., Sun, M., Dong, H. (2008). A first map of tropical Africa's above-ground biomass derived from satellite imagery. Environ. Res. Lett., 3, 1-9. doi:10.1088/1748-9326/3/4/045011

Boyemba, F. (2011). Ecologie de Pericopsis elata (Harms) Van Meeuwen (Fabaceae), arbre de forêt tropicale africaine à répartition agrégée. Thèse de Doctorat, Université Libre de Bruxelles, 206p.

Bussmann, R.W. (2006). Vegetation zonation and nomenclature of African Mountains - An overview. lyonia, 11, 41-66.

Chave, J., Réjou-Méchain, M., Búrquez, A., Chidumayo, E., Colgan, M.S., 
Delitti, W.B.C., Duque, A., Eid, T., Fearnside, P.M., Goodman, R.C., Henry, M., Martínez-Yrízar, A., Mugasha, W. a, Muller-Landau, H.C., Mencuccini, M., Nelson, B.W., Ngomanda, A., Nogueira, E.M., Ortiz-Malavassi, E., Pélissier, R., Ploton, P., Ryan, C.M., Saldarriaga, J.G., Vieilledent, G. (2014). Improved allometric models to estimate the aboveground biomass of tropical trees. Glob. Chang. Biol., 20, 3177-3190. doi:10.1111/gcb.12629

De Cáceres, M. (2013). How to use the indicspecies package (ver. 1.7.1). R Project, 29p.

Djomo, A.N., Ibrahima, A., Saborowski, J., Gravenhorst, G. (2010). Allometric equations for biomass estimations in Cameroon and pan moist tropical equations including biomass data from Africa. For. Ecol. Manage., 260, 1873-1885. doi:10.1016/j.foreco.2010.08.034

Djuikouo, K., Doucet, J., Nguembou, C.K., Lewis, S.L., Sonke, B. (2010). Diversity and aboveground biomass in three tropical forest types in the Dja Biosphere Reserve , Cameroon. Afr. J. Ecol., 48, 1053-1063.

Fayolle, A., Doucet, J.-L., Gillet, J.-F., Bourland, N., Lejeune, P., 2013. Tree allometry in Central Africa: Testing the validity of pantropical multi-species allometric equations for estimating biomass and carbon stocks. For. Ecol. Manage., 305, 29-37. doi:10.1016/j.foreco.2013.05.036

Feldpausch, T.R., Lloyd, J., Lewis, S.L., Brienen, R.J.W., Gloor, M., Monteagudo Mendoza, A., Lopez-Gonzalez, G., Banin, L., Abu Salim, K., Affum-Baffoe, K., Alexiades, M., Almeida, S., Amaral, I., Andrade, A., Aragão, L.E.O.C., Araujo Murakami, A., Arets, E.J.M.M., Arroyo, L., Aymard C., G.A., Baker, T.R., Bánki, O.S., Berry, N.J., Cardozo, N., Chave, J., Comiskey, J.A., Alvarez, E., de Oliveira, A., Di Fiore, A., Djagbletey, G., Domingues, T.F., Erwin, T.L., Fearnside, P.M., França, M.B., Freitas, M.A., Higuchi, N., E. Honorio C., Iida, Y., Jiménez, E., Kassim, A.R., Killeen, T.J., Laurance, W.F., Lovett, J.C., Malhi, Y., Marimon, B.S., MarimonJunior, B.H., Lenza, E., Marshall, A.R., Mendoza, C., Metcalfe, D.J., Mitchard, E.T.A., Neill, D.A., Nelson, B.W., Nilus, R., Nogueira, E.M., Parada, A., Peh, K.S.-H., Pena Cruz, A., Peñuela, M.C., Pitman, N.C.A., Prieto, A., Quesada, C.A., Ramírez, F., Ramírez-Angulo, H., Reitsma, J.M., Rudas, A., Saiz, G., Salomão, R.P., Schwarz, M., Silva, N., Silva-Espejo, J.E., Silveira, M., Sonké, B., Stropp, J., Taedoumg, H.E., Tan, S., ter Steege, H., Terborgh, J., Torello-Raventos, M., van der Heijden, G.M.F., Vásquez, R., Vilanova, E., Vos, V.A., White, L., Willcock, S., Woell, H., Phillips, O.L. (2012). Tree height integrated into pantropical forest biomass estimates. Biogeosciences, 9, 3381-3403. doi:10.5194/bg-9-3381-2012

Feyissa, T., Zhu, L., Negash, L., Welander, M., 2007. Regeneration and genetic transformation of Hagenia abyssinica(Bruce)J.F.Gmel.(Rosaceae) with rolb gene. Plant Cell Tissue Organ Cult, 88, 277-288. doi:http://dx.doi.org/10.1007/s11240-006-9200-5 
Fischer, E. (1993). Taxonomic results of the Bryotrop-Expedition to Zaire and Rwanda ( Description of Collecting Sites . The Vegetation of KahuziBiega-National Park / Zaire , Nyungwe Forest and Virunga volcanoes / Rwanda). Trop. Bryol., 8, 13-37.

Fischer, E. (1996). Die Vegetation Des Parc National De Kahuzi-Biega, SudKivu, Zaire, Franz Steiner Verlag. Edition : Stuttgart, Berlin/Allemagne, 239p.

GIEC (2006). Terres forestières : Agriculture, foresterie et autres affectations des terres. Lignes directrices 2006 du GIEC pour les inventaires nationaux de gaz à effet de serre, 93p.

Habiyaremye, M.K. (1995). Etude phytoécologique de la dorsale orientale du lac Kivu (Rwanda). Thèse de Doctorat, Université Libre de Bruxelles, 276p. Henry, M., Besnard, a., Asante, W. a., Eshun, J., Adu-Bredu, S., Valentini, R., Bernoux, M., Saint-André, L. (2010). Wood density, phytomass variations within and among trees, and allometric equations in a tropical rainforest of Africa. For. Ecol. Manage., 260, 1375-1388. doi:10.1016/j.foreco.2010.07.040

Imani, M. G., Zapfack, L., Kalume, J., Riera, B., Cirimwami, L., Boyemba, F. (2016). Woody vegetation groups and diversity along the altitudinal gradient in mountain forest: Case study of Kahuzi-Biega National Park and its surroundings, RD Congo. Journal of Biodiversity and Environmental Sciences, Submitted

Kabonyi, N.C. (2012). Analyse palynologique de divers sondages du secteur de haute altitude du Parc National de Kahuzi-Biega. Paléoenvironnements et paléoclimats au Pléistocène supérieur et à l’Holocène. Thèse de Doctorat, Université de Liège, 128p.

Kearsley, E., Haulleville, T. De, Hufkens, K., Alidé, K., Benjamin, T., Baert, G., Huygens, D., Kebede, Y., Defourny, P., Bogaert, J., Beeckman, H., Steppe, K., Verbeeck, H. (2013). Conventional tree height-diameter relationships significantly overestimate aboveground carbon stocks in the Central Congo Basin. Nat. Commun., 4, 1-8. doi:10.1038/ncomms3269

Kurz, W.A., Stinson, G., Rampley, G.J., Dymond, C.C., Neilson, E.T. (2008). Risk of natural disturbances makes future contribution of Canada's forests to the global carbon cycle highly uncertain. Environ. Sci., 105, 15511555.

Laporte, N., Baccini, A., Goetz, S., Mekui, P., Bausch, A. (2010). Une première estimation de la biomasse ligneuse aérienne d'Afrique sur la base d'images satellites et d'inventaires forestiers, in: Proceeding of the Conference on Carbon Stock and Fluxes, Comifac. p. 9.

Léonard, E., Oswold, M. (1996). Une agriculture forestière sans forêt. Changements agroclimatiques et innovations paysannes en Côte d'Ivoire. Natures - Sci. - Sociétés, 4, 13. 
Lewis, S.L., Lopez-Gonzalez, G., Sonké, B., Affum-Baffoe, K., Baker, T.R., Ojo, L.O., Phillips, O.L., Reitsma, J.M., White, L., Comiskey, J. a, Djuikouo K, M.-N., Ewango, C.E.N., Feldpausch, T.R., Hamilton, A.C., Gloor, M., Hart, T., Hladik, A., Lloyd, J., Lovett, J.C., Makana, J.-R., Malhi, Y., Mbago, F.M., Ndangalasi, H.J., Peacock, J., Peh, K.S.-H., Sheil, D., Sunderland, T., Swaine, M.D., Taplin, J., Taylor, D., Thomas, S.C., Votere, R., Wöll, H. (2009). Increasing carbon storage in intact African tropical forests. Nature, 457, 1003-1006. doi:10.1038/nature07771

Lovett, J.C., Marshall, A.R., Carr, J. (2006). Changes in tropical forest vegetation along an altitudinal gradient in the Udzungwa Mountains National Park, Tanzania. Afr. J. Ecol., 44, 478-490. doi:10.1111/j.13652028.2006.00660.x

Mangambu, J. (2013). Taxonomie, biogéographie et écologie des Ptéridophyes de l'écosystème forestier des montagnes du Parc National de Kahuzi-Biega à l'Est de la R.D.Congo. Thèse de Doctorat, Université d'Anvers, 494p.

Mutke, J., Sommer, J.H., Kreft, H., Kier, G., Barthlott, W. (2011). Vascular Plant Diversity in a Changing World: Global Centres and Biome-Specific Patterns. Biodivers. Hotspots, 1, 83-96. doi:10.1007/978-3-642-20992-5

Ngueguim, J.R., 2013. Productivité et diversité floristique des ligneux en forêt dense d'Afrique tropicale humide du Cameroun: Sites de Mangombe Bidou. Thèe de Doctorat, Museum National d'Histoire Naturelle, 213p.

Pan, Y., Birdsey, A.R., Houghton, R., Kauppi, P., Kurz, W., Phillips, O.L., Shvidenko, A., Lewis, S.L., Canadell, J.G., Ciais, P., Jackson, R.B., Pacala, S.W., McGuire, A.D., Piao, S., Rautiainen, A., Sitch, S., Hayes, D. (2011). A Large and persistent carbon sink in the world's forests. Sciences (New. York), 333, 988-993.

Pascal, J. (2003). Notions sur les structures et dynamique des forêts tropicales humides. Revue Forestière Française, numéro special 2003, 118130.

Peh, K.S.H., Sonké, B., Lloyd, J., Quesada, C.A., Lewis, S.L. (2011). Soil does not explain monodominance in a Central African tropical forest. PLoS One, 6 (2) : 1-9. doi:10.1371/journal.pone.0016996

Pierlot, R. (1966). Structure et composition de forest denses d'Afrique Centrale, specialement celles du Kivu, Académie Royale des sciences d'Outre mer, Editions Bruxelles, 373p.

Plumptre, A., Davenport, T., Behangana, M., Kityo, R., Eilu, G., Ssegawa, P., Ewango, C., Meirte, D., Kahindo, C., Herremans, M., Peterhans, J., Pilgrim, J., Wilson, M., Languy, M., Moyer, D. (2007). The biodiversity of the Albertine Rift. Biol. Conserv., 134, 178-194. doi:10.1016/j.biocon.2006.08.021

Ren, H.B., Niu, S.K., Zhang, L.Y., Ma, K.P. (2006). Distribution of vascular 
plant species richness along an elevational gradient in the Dongling Mountains, Beijing, China. J. Integr. Plant Biol., 48, 153-160. doi:10.1111/j.1744-7909.2006.00153.x

Seburanga, J., Nsanzurwimo, A., Folega, F. (2014). Status of Hagenia forest in the Parc National des Volcans, Rwanda: A review of historical records. $J$. Ecol. Natutal Environ. 6 (5): 170-182. doi:10.5897/JENE2014.0441

Tassin, J., Derroire, G., Rivière, J. (2004). Gradient altitudinal de la richesse spécifique et de l'endémicité de la flore ligneuse indigène à l'île de $\mathrm{La}$ Réunion (archipel des Mascareignes). Acta Bot. Galla., 151, 181-196.

Torti, S., PD, C., Kursar, T. (2001). Causes and consequences of monodominance in tropical lowland forests. Am. Nat. 157, 141-53. doi:10.1086/318629.

Turneur, I. (2001). The ecology of three in tropical rain forest. Cambridge University Press, http://dx.doi.org/10.1017/CBO9780511542206, 298p.

Vamosi, J.C., Vamosi, S.M. (2005). Present day risk of extinction may exacerbate the lower species richness of dioecious clades. Divers. Distrib., 11, 25-32. doi:10.1111/j.1366-9516.2005.00119.x

White, F. (1993). The AETFAT Chorological Classification of Africa: History, Methods and Applications. Bull. du Jard. Bot. Natl. Belgique, 62, 225-281.

Yamagiwa, J., Basabose, A.K., Kaleme, K., Yumoto, T. (2005). Diet of Grauer's Gorillas in the Montane Forest of Kahuzi, Democratic Republic of Congo. Int. J. Primatol., 26, 1345-1373.

Yuanying, P., Thomas, S., Tianb, D. (2008). Forest management and soil respiration: Implications for carbon sequestration. Environ. Rev., 16, 93-111. Zanne, A.E., Lopez-Gonzalez, G. Coomes, D.A., Ilic, J., Jansen, S., Lewis, S.L., Miller, R.B., Swenson, N.G., Wiemann, M.C., Chave, J. (2009). Global wood density database. Dryad. Identifier: http://hdl.handle.net/10255/dryad.235. 